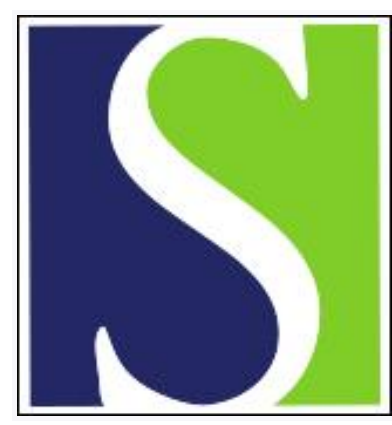

Scand J Work Environ Health 1996;22(3):176-181

https://doi.org/10.5271/sjweh.128

Issue date: Jun 1996

Prevalences of musculoskeletal disorders at the wrist as a function of angles, forces, repetitiveness and movement velocities

by Malchaire JB, Cock NA, Robert AR

The following articles refer to this text: $2001 ; 27(1): 30-40 ; 2001 ; 27$ suppl 1:1-102

Key terms: prevalence; upper limb disorder; work characteristic

This article in PubMed: www.ncbi.nlm.nih.gov/pubmed/8837262

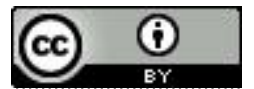




\title{
Prevalence of musculoskeletal disorders at the wrist as a function of angles, forces, repetitiveness and movement velocities
}

\author{
by Jacques B Malchaire, PhD, ${ }^{1}$ Nathalie A Cock, ${ }^{1}$ Annie R Robert, PhD ${ }^{2}$
}

\begin{abstract}
Malchaire JB, Cock NA, Robert AR. Prevalences of musculoskeletal disorders at the wrist as a function of angles, forces, repetitiveness and movement velocities. Scand J Work Environ Health 1996;22:176-81.

Objectives The purpose of this investigation was to study the relationship between the prevalence of musculoskeletal disorders at the wrists and the characteristics of the work conditions in terms of angles, forces, repetitiveness, and movement velocities.

Methods Nine workplaces were selected and the prevalence of wrist disorders was determined by means of a questionnaire for both arms separately, along with characteristics of the 335 subjects (age, weight, height, seniority). A work analysis was performed on subjects selected at random from each workplace by recording, for both wrists during a representative number of work cycles, the angles both in radial and ulnar deviations and in flexion-extension and the surface electromyogram on the hand flexors of the forearm. Repetitiveness (defined as the number of transitions per minute) and movement velocities (in deviation and flexion-extension) were derived from the recordings of the angles.

Results All the derived variables were highly correlated, greater angles and greater forces being associated with greater velocities and higher repetitiveness. A multivariate linear regression model for the prediction of the prevalence of musculoskeletal disorders of the wrist was constructed $(R=0.904)$. Height, weight, seniority, angles in radial-ulnar deviation, and forces were significant and independent predictors of the prevalence.

Conclusions The prevalence of wrist disorders is significantly linked to wrist angles in deviation and to forces exerted. Due to their high correlation with force, the repetitiveness indices and velocities, as defined, do not appear to play an additional role. Further research is needed to find alternative ways of characterizing repetitiveness.
\end{abstract}

Key terms prevalence, upper limb disorders, work characteristics.

Although the scientific evidence still appears to be debatable (1), there is a consensus that repetitiveness, exerted forces, and hand postures are associated with the development of musculoskeletal disorders of the wrist and, in particular, carpal tunnel syndrome (2).

In the present study, disorders were defined according to Kroemer (3): "a collective term for syndromes characterized by discomfort, impairment, disability or persistent pain in joints, muscles, tendons and other soft tissues, with or without physical manifestations, including tenosynovitis, ganglionic cysts, carpal tunnel syndrome, ... [p 274]."

Several studies have attempted to find the relative role played by these factors, to which could be added the movement velocity of the wrist. Silverstein et al $(2,4)$ reported two studies in which jobs were categorized into the following four exposure groups: low force-low repetitiveness, high force-low repetitiveness, low force-high repetitiveness, and high force-high repetitiveness. The first study (4) concerned wrist disorders in general and concluded that force [odds ratio (OR) 4.4] was a more important risk factor than repetitiveness (OR 2.8). The second (2) concerned only carpal tunnel syndrome and concluded the opposite (force OR 2.9 and repetitiveness OR 5.5). The two studies were apparently conducted on the same population and the contradictory results were not discussed.

Marras \& Schoenmarklin $(5,6)$ analyzed the relationship between the development of carpal tunnel syndrome and the amplitudes, velocities, and accelerations

1 Université catholique de Louvain, Unité Hygiène et Physiologie du Travail (Catholic University of Louvain, Work Physiology and Hygiene Unit), Bruxelles, Belgium.

2 Université catholique de Louvain, Institut de Statistique (Catholic University of Louvain, Institute of Statistics), Louvain, Belgium.

Reprint requests to: Dr JB Malchaire, Université catholique de Louvain, Unité Hygiène et Physiologie du Travail, Clos Chapelle-aux-Champs, 3038, B - 1200 Bruxelles, Belgium. 
of movements. They concluded that velocities and accelerations in flexion-extension were the best predictors. They did not consider the force variables.

Lin et al (7) conducted an experimental study in which three subjects performed repetitive flexing of the wrist at two different paces for $1 \mathrm{~h}$, against two levels of force and at two angles. The discomfort was rated at the end of the experiment on a $10-\mathrm{cm}$ linear analog scale from "no" to "very high." This discomfort was very significantly correlated with the three parameters $(\mathrm{R}=0.980)$, and weighting factors were proposed for each factor. These results, as well as those gained from experimental studies, definitely depend upon the range of the paces, forces, and angles investigated and are therefore of very little quantitative significance. In addition, discomfort might not be strictly correlated with the physiological effects.

The relative influence of the different factors concerning the development of upper limb disorders can therefore be determined only from field studies in which the prevalence of upper limb disorders, the characteristics of the work conditions in terms of angles, forces, repetitiveness and velocities, and finally all the potential confounders such as age, weight, and seniority would be quantitatively determined concurrently. This was the purpose of the present study.

\section{Study population}

Three hundred and thirty-five workers were interviewed by a physiotherapist, and data concerning age, weight, height, and seniority were recorded. In addition, the subjects were asked whether they had suffered from musculoskeletal disorders at the right and left wrists during the last 12 months (either yes or no). Additional information was collected concerning the nature, the duration, and the frequency of these disorders, but it has not been reported in this communication.

The 335 workers came from nine workplaces, involving different levels of force, repetitiveness, and wrist angular postures. They are the following (with the prevalences of the disorders in the right and left wrists respectively):

1. 35 men performing various tasks, such as managing claims, and answering the phone in an insurance company $(2.9 \%, 0.0 \%)$;

2. 34 women in the same company but performing more administrative tasks: data encoding, secretarial work, and the like $(8.8 \%, 6.1 \%)$;

3. 21 men working on computers, editing and managing clients' data in a bank $(28.6 \%, 4.8 \%)$;

4. 63 women in the same bank, encoding check data at a high speed $(20.6 \%, 9.5 \%)$;
5. 17 women sewing seat covers in an automobile assembly plant $(-, 5.9 \%)$;

6. 25 women packing in a pastry plant $(16.0 \%, 4.0 \%)$;

7. 37 men in the same plant, preparing the paste decorating the pastries manually $(16.2 \%, 8.1 \%)$;

8. 84 men assembling seats in a car plant $(39.3 \%$, $34.5 \%)$;

9. 19 meat cutters $(42.1 \%, 21.1 \%)$.

Table 1 gives the mean age, weight, height, and seniority of the whole group of workers.

Work analyses were performed on a total of 100 workers, that is, on about 11 subjects selected at random from each workplace from a list of all the workers at each workplace. Table 1 also gives the mean characteristics for this sample. Using a Student t-test for independent samples, it was checked that the group of 100 workers was an unbiased sample of the whole group of 335 workers. Data comparison within each workplace showed the same similarities between the two groups.

\section{Methods}

The work analyses involved the recording, for both arms separately, of the angles in radial deviation, ulnar deviation, flexion, and extension by means of goniometers (Penny \& Giles, Blackwood Ltd, United Kingdom). In addition, electromyographic (EMG) surface electrodes (Medicotest N-00-S) were placed on the hand flexors of both forearms, and the root mean square (RMS) EMG signal was continuously recorded using a Mega ME 3000 muscle tester (Mega Electronics Ltd, Kuopio, Finland). The observation lasted $45 \mathrm{~min}$ (workplaces 1,2 , 3,4 ) or $60 \mathrm{~min}$ ( 3 work cycles for workplace 5,4 cycles for workplace 8 , and 6 cycles for workplaces 6 and 7) or up to 90 min (for workplace 9 - meat cutting). Prior to the recording at the workplace, the maximum angles were determined in the four directions and for both wrists. The subjects also performed three maximum voluntary grasp efforts with both hands (during $10 \mathrm{~s}$ with a rest of $2 \mathrm{~min}$ in between) on a hand dynamometer (JAMAR PC 5030G1 by Camp Ltd, United Kingdom)

Table 1. Mean characteristics with standard deviations for the two groups of workers.

\begin{tabular}{|c|c|c|c|c|}
\hline & \multicolumn{2}{|c|}{$\begin{array}{l}\text { Group } A^{a} \\
(N=335)\end{array}$} & \multicolumn{2}{|c|}{$\begin{array}{l}\text { Group } B^{b} \\
(N=100)\end{array}$} \\
\hline & Mean & SD & Mean & SD \\
\hline Age (years) & 36.5 & 8.9 & 37.7 & 7.0 \\
\hline Weight (kg) & 71.4 & 14.6 & 70.3 & 15.7 \\
\hline Height (cm) & 170.0 & 9.3 & 169.7 & 8.9 \\
\hline Seniority (years) & 11.3 & 8.7 & 12.5 & 8.3 \\
\hline
\end{tabular}

a Characteristics of the total population.

b Characteristics of the groups of workers who were examined during the work analyses. 
and the corresponding average maximum EMG value was recorded.

The angular signals were recorded using a modulated frequency (FM) recorder (TEAC HR-30G, TEAC Corp, Japan) with a linear frequency response of up to $100 \mathrm{~Hz}$ and a dynamic range of $32 \mathrm{~dB}$. They were analyzed in the laboratory to determine the instantaneous angles in radial deviation, ulnar deviation, flexion, and extension at any time. These angles were divided by the corresponding maximum individual angles to determine the instantaneous relative angles in the four directions. For each work analysis, the mean relative angles in deviation (either ulnar or radial) and in the other plane (either flexion or extension) were calculated. Maximum acceptable angle values of $50 \%$ in deviation and $60 \%$ in flexion-extension were adopted, and the percentages of the time the instantaneous relative angles exceeded these limits were determined in each plane. These results were summarized in the following four variables for each work analysis and, finally, across these work analyses, for each workplace and each wrist: $\mathrm{mDr}$ : mean relative angle in radial or ulnar deviation (in \%); mFr: mean relative angle in flexion or extension (in \%); $\mathrm{pD} \%$ : percentage of the time the relative angle in radial or ulnar deviation exceeded $50 \%$ of the maximum angles; and $\mathrm{pF} \%$ : percentage of the time the relative angle in flexion or extension exceeded $60 \%$ of the maximum angles.

An index of repetitiveness was first derived from the recordings of the angles $\left(R_{\text {ang }}\right)$ as the number of angular transitions (per minute) of the wrist from a "neutral" position to an "extreme" position (more than $50 \%$ of the maximum angle in deviation or more than $60 \%$ in flexion-extension). Again, each workplace and each wrist was characterized by the mean repetitiveness computed from the individual work analyses.

In addition, the angular signals were derived to determine the movement velocity in each plane; mVd and $m V f$ were computed as the mean absolute velocities in degrees per second in deviation and flexion-extension for each work analysis and finally for each workplace and wrist.

The mean relative RMS EMG value (mEMGr) was computed as the percentage of the maximum EMG value initially recorded. A limit value of $15 \%$ was adopted for the relative EMG and the percentage of the time this limit was exceeded ( $\mathrm{pEMG} \%$ ) was computed.

A final expression of repetitiveness $\left(R_{t o t}\right)$ was computed as the number of transitions per minute from a "neutral" condition to an "extreme" condition with the relative angles greater than $50 \%$ in deviation or $60 \%$ in flexion-extension, or with the relative EMG greater than $15 \%$.

For all the workplaces and the two wrists, a data base was formed with the prevalence of wrist disorders and the mean characteristics (age, weight, height, seniority), (for the 335 workers) and the angles, forces, repetitiveness, and velocities averaged for all the work analyses at each workplace. Linear correlation coefficients were computed for each pair of parameters, and univariate and multivariate (backward stepwise) linear regression analyses were performed with the prevalence of wrist disorders as the dependent variable and all other factors as independent variables.

\section{Results}

Table 2 gives the means and standard deviations of the variables derived from the work analyses and averaged for each workplace and each wrist (17 conditions, as data for the right wrist were missing for workplace number 5).

The rather large standard deviations indicated large and indeed significant differences between the 17 conditions. All these variables were however highly correlated, as indicated by the correlation matrix reported in table 3 . While correlations between variables from the same origin ( $\mathrm{mFr}$ and $\mathrm{R}_{\text {ang }}$, for instance) are obvious, it is

Table 2. Means and standard deviations of the variables of the work analyses $(N=100) .(m D r=$ relative angle in deviation, $\mathrm{mFr}=$ relative angle in flexion-extension, $\mathrm{mEMGr}=$ relative $\mathrm{EMG}$, $\mathrm{pD} \%=$ percentage of time relative deviation angle $>50 \%$, $\mathrm{pF} \%=$ percentage of time relative flexion-extension angle $>60 \%$, pEMG $\%=$ percentage of time relative $E M G>15 \%, R_{\text {ang }}=$ angular repetitiveness, $R_{t o t}=$ angular or force repetitiveness, $\mathrm{mVd}=$ mean velocity in deviation, $\mathrm{mVf}=$ mean velocity in flexion-extension, $E M G$ = electromyography)

\begin{tabular}{lcr}
\hline Variables & Mean & SD \\
\hline $\mathrm{mDr}(\%)$ & 42.7 & 6.3 \\
$\mathrm{mFr}(\%)$ & 36.2 & 8.3 \\
$\mathrm{mEMGr}(\%)$ & 20.4 & 10.3 \\
$\mathrm{pD} \%$ & 32.5 & 7.5 \\
$\mathrm{pF} \%$ & 12.5 & 8.3 \\
$\mathrm{pEMG} \%$ & 32.4 & 20.3 \\
$\mathrm{R}_{\text {ang }}\left(\mathrm{N} \cdot\right.$ min $\left.^{-1}\right)$ & 15.1 & 3.9 \\
$\mathrm{R}_{\text {tot }}\left(\mathrm{N} \cdot\right.$ min $\left.^{-1}\right)$ & 21.3 & 6.4 \\
$\mathrm{mVd}\left(\right.$ degrees $\left.^{-1} \cdot \mathrm{s}^{-1}\right)$ & 29.7 & 6.3 \\
$\mathrm{mVf}\left(\right.$ degrees $\left.\cdot \mathrm{s}^{-1}\right)$ & 43.6 & 11.1 \\
\hline
\end{tabular}

Table 3. Correlation coefficient $\left(10^{-2}\right)$ matrix between seven variables from the work analyses. ( $\mathrm{mDr}=$ relative angle in deviation, $\mathrm{mFr}=$ relative angle in flexion-extension, $\mathrm{mEMGr}=$ relative $\mathrm{EMG}$ signal, $R_{\text {ang }}=$ angular repetitiveness, $R_{\text {tot }}=$ angular and force repetitiveness, $m V d=$ mean velocity in deviation, $m V f=$ mean velocity in flexion-extension, $E M G$ = electromyography)

\begin{tabular}{lrrrrrrr}
\hline Variable & $\mathrm{mDr}$ & $\mathrm{mFr}$ & $\mathrm{mEMGr}$ & $\mathrm{R}_{\text {ang }}$ & $\mathrm{R}_{\text {tot }}$ & $\mathrm{mVD}$ & $\mathrm{mVF}$ \\
\hline $\mathrm{mDr}$ & 100 & -57 & 27 & 49 & 46 & 47 & 14 \\
$\mathrm{mFr}$ & -57 & 100 & -55 & -61 & -60 & -86 & -60 \\
$\mathrm{mEMGr}$ & 27 & -55 & 100 & 86 & 85 & 70 & 86 \\
$\mathrm{R}_{\text {ang }}$ & 49 & -61 & 86 & 100 & 97 & 82 & 87 \\
$\mathrm{R}_{\text {tot }}$ & 46 & -60 & 85 & 97 & 100 & 82 & 89 \\
$\mathrm{mVd}$ & 47 & -86 & 70 & 82 & 82 & 100 & 80 \\
$\mathrm{mVf}$ & 14 & -60 & 86 & 87 & 89 & 80 & 100 \\
\hline
\end{tabular}


worth noting the rather strong correlation between $\mathrm{mEMGr}$, the repetitiveness indices, and the velocities.

Univariate correlation analyses showed that the prevalences of wrist disorders were significantly and positively correlated with the mean values of the forces ( $m E M G r$ and $p E M G \%)$, the repetitiveness $\left(R_{\text {ang }}\right.$ and $\left.R_{t o t}\right)$, the angles (mDr), and the velocity ( $\mathrm{mVf}$ ), as well as with the average weight and height.

Table 4 gives the coefficients (and their significance) of the multivariate linear regression model for the prediction of the prevalence $(R=0.904)$. None of the other variables of the work conditions nor of the cross products (interactions) gave an additional significant contribution to the prevalence prediction.

It is interesting to note that, for a group of average workers (170 cm and $70 \mathrm{~kg}$ regardless of gender), after five years of exposure to work conditions characterized by an $\mathrm{mDr}$ equal to $50 \%$ and an $\mathrm{mEMGr}$ equal $15 \%$, the prevalence of wrist disorders would be roughly zero.

\section{Discussion}

The variables of force and angles were expressed in terms of both their mean relative values and the percentage of the time they exceeded a limit value. For the angles, absolute limit values have been proposed by Armstrong (8): 9 degrees for radial deviation, 19 degrees for ulnar deviation, and 45 degrees for flexion and extension. In order to take into consideration the very large interindividual differences in maximum angles, we chose to adopt relative limit values, in percentages of the maximum angles. According to the data published by Hoppenfeld \& Hutton (9), the average values of the maximum angles can be taken as 20,30,80 and 70 degrees so that limit values of $50 \%$ in radial-ulnar deviation and $60 \%$ in flexion-extension can be adopted. Similarly, a limit value of $15 \%$ was adopted for the relative EMG value; a consensus seems to exist indeed to adopt this percentage of maximum strength as the level below which fatigue would not develop $(2,10)$. This limit can be compared with the limit values proposed by Byström (11) for continuous isometric contractions [10\% of maximal voluntary contraction (MVC)] and intermittent contractions (17\% MVC). It must be kept in mind however that the relative EMG value measured using surface electrodes does not only represent the force exerted by the finger flexors, as it is also influenced by the efforts in wrist flexion. Besides, Duque et al (12) showed how this EMG value is also strongly influenced by the hand flexion angle. A limit value of $15 \%$ for mEMGr therefore very likely means a lower limit for the exerted force of the finger flexors.

Repetitiveness can be defined on the basis of the cycle time or biomechanically as the number of move-
Table 4. Coefficients (and significance) of the multivariate linear regression model for the prediction of the prevalence of wrist disorders. $(\mathrm{mDr}=$ relative angle in deviation, $\mathrm{mEMGr}=$ relative $\mathrm{EMG}$ signal)

\begin{tabular}{lcc}
\hline Variable & Coefficient & P-value \\
\hline Constant & -435.8 & 0.01 \\
Height $(\mathrm{cm})$ & 2.90 & 0.02 \\
Weight $(\mathrm{kg})$ & -2.23 & 0.03 \\
Seniority (years) & 2.53 & 0.01 \\
mDr $(\%)$ & 1.17 & 0.02 \\
mEMGr (\%) & 1.87 & 0.00 \\
\hline
\end{tabular}

ments per unit of time. Silverstein et al (2) adopted the first method and defined highly repetitive jobs as "those with a cycle time of less than 30 seconds or more than $50 \%$ of the cycle time involved performing the same kind of fundamental cycles [p 344]." According to this criterion, which indeed is very simple to use, all the work conditions considered in the present study are highly repetitive. The second method was therefore adopted to differentiate between the conditions, and a repetitiveness index was defined for the movements only $\left(R_{\text {ang }}\right)$, as well as for the angles or forces $\left(R_{\text {tot }}\right)$ using, as criteria, the number of transitions from a "neutral" to an "extreme" condition, the threshold values being the ones discussed. While these indices appear to reflect repetitiveness at the workplace, they have two major drawbacks. They are difficult to compute, and they are highly correlated with the variables describing the movements ( $\mathrm{mDr}$ and $\mathrm{mFr}$ ) and the forces (mEMGr). The observed correlation coefficient between $R_{\text {ang }}$ and mEMGr was 0.86 . Therefore the use of these indices is drastically limited.

The same appears to be true for the movement velocities definitely correlated with the mean force (mEMGr): 0.702 for $\mathrm{mVd}$ and 0.864 for $\mathrm{mVf}$. It clearly appears that jobs requiring more force of the wrist will be performed with higher repetitiveness and higher velocities. It is interesting to note that all the correlation coefficients in table 3 are positive with the exception of those related to the mean relative angle in flexion-extension ( $\mathrm{mFr}$ ). A more-detailed graphic analysis (not described) showed that this is due to the fact that, for the first four workplaces involving mostly administrative work, the workers systematically adopted a static posture of the wrists with angles greater than 25 degrees in extension and little motion, while the other workplaces were characterized by lower angles on the average but also by frequent movements of large amplitude.

As all the variables describing the work conditions were highly correlated, it is difficult to determine the role played by the repetitiveness and velocities in the development of wrist disorders.

Table 4 shows that mean values are better predictors of the prevalence of wrist disorders than the percentages 
of time above limits, as they do not appear in the final regression model.

This, however, cannot be a definite conclusion, as these last variables depend strongly upon the limits adopted. Further research must be conducted in this direction, as it appears reasonable to think that only large values of angles or force are detrimental. An attempt was made to take into account the means plus one standard deviation for each variable, these being obviously more representative of the large values encountered at the workplaces, rather that the mean values. This procedure led to a model with the same variables as in table 4 but with a lower regression coefficient.

As mentioned previously, all the variables were highly correlated, and the prediction equation should be interpreted accordingly. Weight and height were strongly correlated $(R=0.945)$ and the negative coefficient of the weight should therefore be considered as a counterbalance to the large and positive coefficient of the height.

Among the five variables of the prediction model, mEMGr also appeared to be strongly correlated with seniority $(R=-0.851)$ suggesting that people with greater seniority - and older — exert less force. A possible explanation could be that workers with greater seniority are more skilled and perform the same work with less energy. It could also be, however, that the less demanding workplaces are occupied, by mere chance or autoselection, by older people.

When seniority was removed from the model, the coefficient of $\mathrm{mEMGr}$ became 0.85 instead of 1.87 , clearly indicating the magnitude of the counterbalancing effect on seniority.

The variables $\mathrm{mDr}$ and $\mathrm{mEMGr}$ were, on the contrary, rather poorly correlated $(R=0.267)$, and their effect in the prediction of wrist disorders can therefore be considered roughly additive. It is worth noting that the mean amplitude of movement in flexion-extension ( $\mathrm{mFr}$ ) did not directly appear in the prediction model, possibly because of correlations between $\mathrm{mFr}$ and $\mathrm{mEMGr}(\mathrm{R}=$ $-0.55)$ or $\mathrm{mDr}(\mathrm{R}=-0.57)$. These correlations, although statistically significant, were not strong, however, and this observation can be linked to that of Marras \& Schoenmarklin (5), according to whom deviation angles were more important than angles in flexion-extension but they appeared to be less robust indicators than velocity, especially in flexion-extension.

From this prediction model, as well as from the strong correlations between the variables of the work conditions, it can be concluded that the most significant factor on which to act in order to decrease the probability of wrist disorders is the force exerted; the second is the angles in radial-ulnar deviation. Reducing force has indeed a strong influence on reducing the velocity of movement, the amplitude of movement in flexion and extension, and the angular repetitiveness. An ergonomic rede- sign of the tools to avoid the movements in deviation can also prove to be profitable.

As presented earlier, the limit values of $50 \%$ for the $\mathrm{mDr}$ and $15 \%$ for the mEMGr are based on data from the literature. The present study does not make possible the validation of these limits, nor was it designed to validate these limits separately. However, the fact that, for a group of average workers (in height, weight and seniority), the predictive prevalence is zero when the $\mathrm{mDr}$ and $\mathrm{mEMGr}$ are equal to these values strongly suggests that these two figures are valid as threshold limit values, for, respectively, the mean relative angles in deviation and the mean relative forces (as measured by means of the surface EMG).

\section{Acknowledgments}

This research was partly supported by a grant from the Federal Services for Scientific, Technical and Cultural Affairs from the Belgian Federal State.

The authors want to thank the occupational physicians and the workers who participated in this study.

\section{References}

1. Stock SR. Workplace ergonomic factors and the development of musculoskeletal disorders of the neck and upper limbs: a meta-analysis. Am J Ind Med 1991;19:87-107.

2. Silverstein BA, Fine LJ, Armstrong TJ. Occupational factors and carpal tunnel syndrome. Am J Ind Med 1987;11:343-58.

3. Kroemer KHE. Cumulative trauma disorders: their recognition and ergonomics measures to avoid them. Appl Ergon $1989 ; 20: 274-80$.

4. Silverstein BA, Fine LJ, Armstrong TJ. Hand wrist cumulative trauma disorders in industry. Br J Ind Med 1986;43:77984.

5. Marras WS, Schoenmarklin RW. Wrist motions and CTD risk in industrial and service environments. In: Quéinnec Y, Daniellou $F$, editors. Designing for everyone: proceedings of the 11 th Congress of the International Ergonomics Association. London: Taylor and Francis, 1991:36- -8 .

6. Marras WS, Schoenmarklin RW. Wrist motions in industry. Ergonomics 1993;36:341- 51.

7. Lin ML, Radwin RG, Snook SH. Development of a relative discomfort profile for repetitive wrist motions and exertions. In: International Ergonomics Association. Ergonomics in occupational health and safety: proceedings of the 12th Congress of the International Ergonomics Association. Toronto: Human Factors Association of Canada, 1994:219-21.

8. Armstrong TJ. Upper-extremity posture: definition, measurement and control. In: Corlett N, Wilson J, Manenica I, editors. The ergonomics of working postures. London: Taylor and Francis, 1986:59-73.

9. Hoppenfeld $\mathrm{S}$, Hutton R. Examen clinique des membres et du rachis. Paris: Masson, 1984 
10. Putz-Anderson V. Cumulative trauma disorders - a manual for musculoskeletal diseases of the upper limbs. London: Taylor and Francis, 1988.

11. Byström S. Physiological response and acceptability of isometric intermittent handgrip contractions. Stockholm: Arbe- tarskyddsverket 1991:1-82. Arbete och Hälsa 38.

12. Duque J, Masset D, Malchaire J. Evaluation of handgrip force from EMG measurements. Appl Ergon 1995;36:61-6.

Received for publication: 5 April 1995 